\title{
Primary Non-Hodgkin's Lymphoma of the Eyelid
}

\author{
Sonia Chhabra, Sunita Singh, Rajnish Kalra, Amrita Duhan, Nisha Marwah, Rajeev Sen \\ Department of Pathology, Pandit Bhagwat Dayal Sharma Post Graduate Institute of Medical \\ Sciences, Rohtak, India
}

\begin{abstract}
Malignant tumours affecting the eyelid include basal cell carcinoma (85\% to 95\%), squamous cell carcinoma (approximately 5\%), sebaceous carcinoma (1\% to 5\%), and malignant melanoma (1\%). Non-Hodgkin's lymphoma of the eyelid is rare. This report is of an 80-year-old woman who presented with diffuse left upper eyelid swelling, clinically suspected to be a haemangioma. Rare diseases should be considered in differential diagnoses because they have the potential for rapid systemic involvement and may need treatment that is different from that of other common diseases at the same site.
\end{abstract}

Key words: Eyelids, Lymphoma, non-Hodgkin

Asian J Ophthalmol. 2011;12:183-5.

\section{Introduction}

Lymphomas can arise from any lymphatic tissue (lymph nodes, spleen, thymus gland, or mucosa [mucosa-associated lymphoid tissue; MALT]). The eyelid is a rare site for lymphoma, but a few isolated cases have been reported. ${ }^{1}$ These lymphomas should not be confused with other cancers that can affect the eyelid such as melanoma and sebaceous carcinoma, including mesenchymal tumours (Kaposi sarcoma), ${ }^{1}$ all of which have different modes of treatment. This report is of lymphoma of the eyelid in an 80-yearold woman, who presented with left upper eyelid swelling, clinically suspected to be a haemangioma.

\section{Case report}

An 80-year-old woman presented to the Outpatient Department at the Pandit Bhagwat Dayal Sharma Post Graduate Institute of Medical Sciences, Rohtak, India, in 2010 with left upper eyelid swelling (Figure 1). At examination, there was diffuse reddish blue enlargement of the left upper eyelid, involving the entire length of the lid, obscuring the eyeball. The swelling was soft to firm in consistency, non-tender, and had progressed rapidly to the presenting size of $3 \times 3 \mathrm{~cm}$ within 20 days.

Ocular examination of the left eye could not be done, whereas the right eye had mature cataract and a normal fundus. Clinically, a diagnosis of haemangioma was made and fine-needle aspiration cytology was advised. Fine-needle aspiration yielded bloodmixed aspirate. Microscopic examination showed a monomorphic

Correspondence: Dr Sonia Chhabra, House No 1173/23, DLF Colony, Rohtak 124001, India

Tel: (91 093) 1564 7492;

E-mail: dr.soniachhabra@gmail.com population of lymphoid cells. These cells had round to irregular nuclei with granular nuclear chromatin and conspicuous nucleoli in some. Cytoplasm was moderate in amount, basophilic, and fragile. Numerous mitotic figures were also seen. Cytologically, diagnosis of malignant small round cell tumour possibly non-Hodgkin's lymphoma was suggested (Figure 1). A surgical biopsy was advised to confirm the diagnosis.

Figure 1. An 80-year-old woman with (a) diffuse enlargement of left upper eyelid, and (b) fine-needle aspiration cytology showing a monomorphic population of atypical lymphoid cells (Leishman stain; original magnification, x 400).
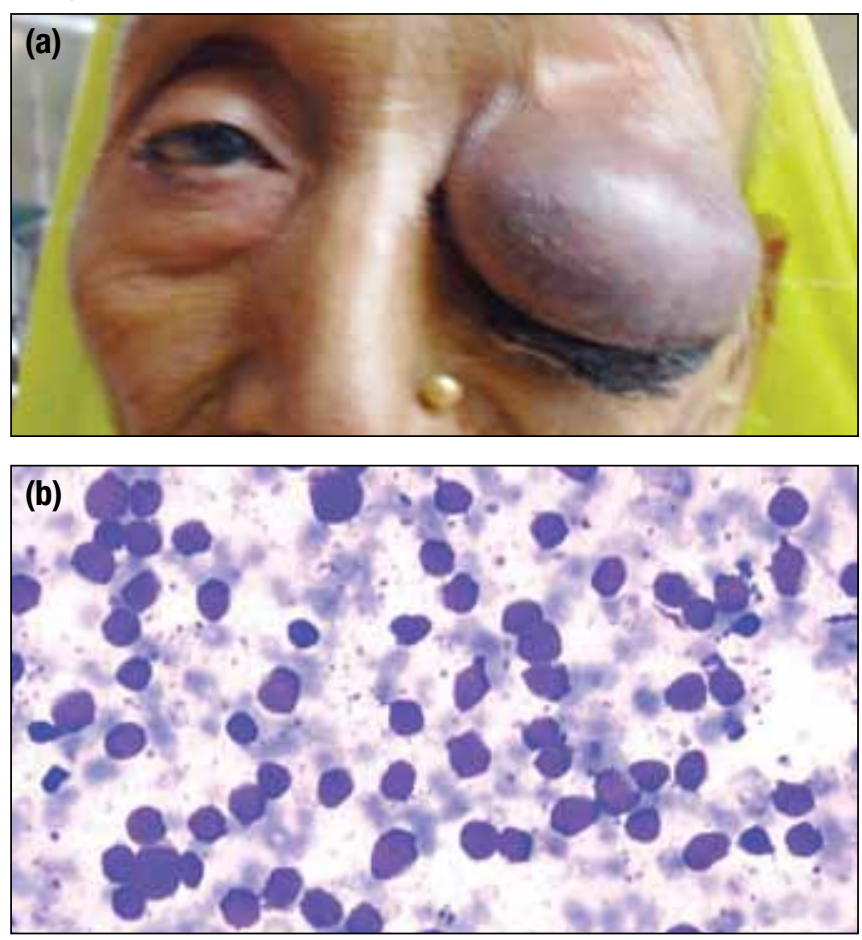
On further investigation, routine blood counts were normal. There was no obvious lymphadenopathy. Chest radiograph, ultrasonography of the abdomen (including the liver and spleen), and complete biochemistry examination were within normal limits. Ultrasonography of the lesion revealed a hypoechoic lesion of $3.2 x$ $1.1 \mathrm{~cm}$, showing flow on colour Doppler imaging. The eyeball and posterior segment of the eye were normal. Computed tomography scan showed a well-defined iso- to hyperdense lesion in the preseptal region of the left orbit displacing the left globe posteriorly, inferiorly, and medially. Fat planes were lost in the anterolateral wall of the left globe. There was no underlying bone defect, calcification or necrosis. Bone marrow failed to show any systemic involvement.

The patient underwent surgical biopsy of the mass, which was sent for histopathological examination. Grossly, multiple grey-white pieces measuring $2 \times 2 \mathrm{~cm}$ in total were received. Microscopy showed dense infiltration by large lymphoid cells in the dermis, with an overlying zone of uninvolved epidermis. These cells had vesicular nuclei, prominent nucleoli, and relatively abundant cytoplasm. Numerous atypical mitoses were also seen (Figure 2). By immunohistochemistry, the large atypical lymphoid cells stained positively for CD45, CD20, and CD10 (Figure 2), and negatively for $\mathrm{CD} 5$, bcl-1, and bcl-2. A histopathological diagnosis of diffuse large $B$ cell lymphoma was made.

\section{Discussion}

B cell lymphoma is a systemic malignancy of lymphoid tissue. Lymphoma of the ocular adnexa (conjunctiva, orbit, and lacrimal gland) represent approximately $8 \%$ of extranodal lymphomas, and eyelid involvement is usually due to forward extension of the tumour. ${ }^{2}$ Most primary orbital adnexal lymphomas are believed to

Figure 2. Microscopy and immunohistochemistry sections showing dense infiltration by atypical lymphoid cells in the dermis with normal overlying epidermis (a) haematoxylin and eosin stain; original magnification, $x$ 20; (b) haematoxylin and eosin stain; original magnification, $x$ 400; (c) the cells were positive for CD10; and (d) CD20 (immunohistochemical staining, $x$ 400).
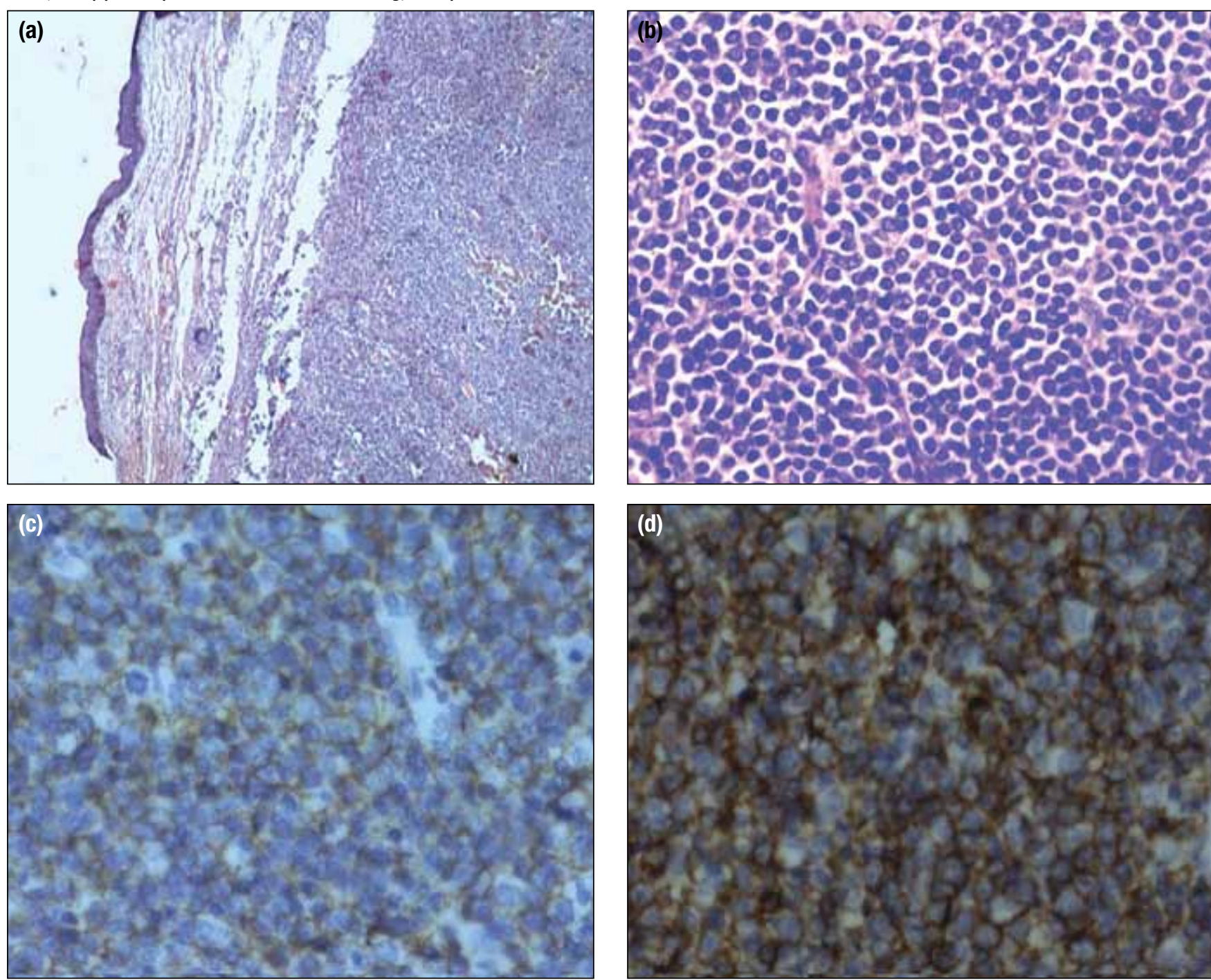
be MALT-type tumours arising from extranodal mucosal tissues, and they may be associated with acquired immunodeficiency disease. Approximately $5 \%$ of patients with systemic lymphoma develop orbital or adnexal metastasis. ${ }^{3}$ Most primary ocular adnexal lymphomas are low-grade lymphomas with marginal zone lymphoma being the most common type. High-grade B-cell lymphomas only occasionally involve the ocular adnexa and T-cell lymphoma, natural killer-cell lymphoma, and Hodgkin's lymphoma are rarely encountered in this site. ${ }^{4}$

Isolated non-Hodgkin's lymphoma of the eyelid is rare. Most non-Hodgkin's lymphoma in the head and neck region arise from Waldeyer's ring and neck lymph nodes. Most of the reported eyelid lymphomas are extranodal marginal zone lymphomas, which are composed of small cells with an occasional large blast surrounding reactive B-cell follicles. The cytology of the tumour cells resembles centrocytes, monocytoid B cells, plasma cells, or small lymphocytes. The tumours are positive for bcl-2, and these tumours have a very indolent course. ${ }^{5}$ Ocular lymphomas are associated with inherited and acquired immunodeficiency diseases, autoimmune diseases such as Sjögren's syndrome, rheumatoid arthritis, and systemic lupus erythematosis. Non-Hodgkin's lymphoma has been linked to exposure to phenytoin, digoxin, and radiation (therapeutic and nuclear energy), and infection with Epstein-Barr virus and hepatitis $C$ virus. Occupational associations include farming and welding. Non-Hodgkin's lymphoma has also been associated with orbital xanthogranuloma, Addison's disease, and Kaposi sarcoma. ${ }^{6}$ The clinical onset is typically insidious and is usually painless. However, as this patient had an aggressive large cell lymphoma, the tumour progressed rapidly. Eyelid involvement presents as swelling, mechanical ptosis, and presence of a subcutaneous mass. ${ }^{3}$

An important differential diagnosis to be considered is pseudolymphoma (lymphoid hyperplasias). Histologically, in these lesions, the pattern is nodular or diffuse, is usually confined to the upper dermis, the so called 'top heavy' pattern, and is frequently associated with germinal centres. The immunophenotype is polymorphous, including both polyclonal B cells, T-helper cells and, rarely, CD30+ cells. ${ }^{7}$

Patients with primary non-Hodgkin's lymphoma of the eyelid, as with other non-Hodgkin's lymphomas, must undergo full screening investigations to determine the extent of disease, select a treatment, and predict the prognosis. Surgical excision is not usually recommended because tumour microinfiltration into the surrounding tissue may be present. However, biopsy is required to establish a diagnosis and for subtyping the lesion. ${ }^{8}$ Patients with low-grade lymphomas are treated with local radiotherapy whereas those with high-grade lymphomas require single-agent or combination chemotherapy. It has been noticed that a more aggressive treatment regimen has not clearly changed the overall survival rate. Immunotherapy for treating non-Hodgkin's lymphoma is showing promising results. ${ }^{9}$

In conclusion, when confronted with an unusual eyelid mass, non-Hodgkin's lymphoma should be considered and a diagnostic biopsy should be performed. This is essential because the diagnosis changes the treatment modality; surgical excision usually performed for more common tumours of the eyelid is not successful in such patients. If not diagnosed correctly, lymphoma of the eyelid may lead to dissemination of the disease. Patients who present with isolated orbital disease should be re-examined periodically for systemic disease.

\section{References}

1. Lymphoma Information Network. Lymphoma of the eyelid. Available from: http://www.lymphomainfo.net/nhl/types/eyelid.html Accessed 21 April 2011.

2. Sarah EC, Krause L, Delecluse HJ, et al. Lymphoproliferative lesions of the ocular adnexa: analysis of 112 cases. Ophthalmology. 1998;105:1430-41.

3. Dutton JJ, Gayre GS, Proia AD, editors. Diagnostic atlas of common eyelid disease. 1st ed. New York: Informa Healthcare; 2007. p 192.

4. Ferry JA, Fung CY, Zukerberg L, et al. Lymphoma of the ocular adnexa: a study of 353 cases. Am J Surg Pathol. 2007;31:170-84.

5. Sharara N, Holden JT, Wojno TH, Feinberg AS, Grossniklaus HE. Ocular adnexal lymphoid proliferations: clinical, histologic, flow cytometric, and molecular analysis of forty three cases. Ophthalmology. 2003;110:1245-54.

6. Al Bermani AM, Talbot EM. Non Hodgkin's lymphoma of the eyelid. CME J Ophthalmol 2000;4:50-1.

7. Fletcher CDM, editor. Diagnostic histopathology of tumors. 3rd ed. Philadelphia: Churchill Livingstone Elsevier; 2007. p 1496-7.

8. Baldini L, Blini M, Guffanti A. Treatment and prognosis in a series of primary extranodal lymphomas of the ocular adnexa. Ann Oncol. 1998;9:779-81.

9. Rosado MF, Byme GE Jr, Ding F. Ocular adnexal lymphoma: a cliniopathological study of a large cohort of patients with no evidence for an association with chlamydia psittaci. Blood. 2006;107:467-72. 